\title{
Study on rapid screening technology of antisense oligonucleotides for SARS-CoV-2
}

Huawei Shen ( $\square$ whs913222@163.com )

Fujian Animal Husbandry General Station https://orcid.org/0000-0002-9259-1404

\section{Short Report}

Keywords: Antisense oligonucleotides, SARS-CoV-2

Posted Date: July 14th, 2021

DOI: https://doi.org/10.21203/rs.3.rs-479520/v2

License: (9) This work is licensed under a Creative Commons Attribution 4.0 International License. Read Full License 


\section{Abstract}

According to the structural characteristics of SARS-CoV-2 nucleic acid,SARS-CoV-2 genomes were virtually segmented.After comparing with human genome,44707 SARS-CoV-2 genomes and 26 primates' genomes, 19 antisense oligonucleotides sequences were selected.Experimental results show that the combined inhibitory effect reaches $100 \%$.

Significance statement囚Currently, effective methods of controlling the spread of SARS-CoV-2 are very limited, only physical isolation and vaccination.Although physical isolation can achieve some short-term results, but can not fundamentally solve the problem of epidemic spread. The time between the development of a vaccine and its actual use is very long.It often happens that the vaccine is developed successfully, but the epidemic situation is beyond control.If a mutant strain emerges, it will take longer.I hope that the rapid screening technology of SARS-CoV-2 antisense oligonucleotides will light up new hope for the future of mankind and contribute oriental wisdom to the world.

\section{Materials}

1.1.Human genome(Download from NCBI),44707 SARS-CoV-2 genomes (Download from NCBI), 26 primates' genomes (Download from Ensembl) .

1.2.Primates:Mouse Lemur,Olive baboon,Orangutan,Pig-tailed macaque,Sooty mangabey,Tarsier,Ugandan red Colobus,Vervet-AGM,Gelada,Gibbon,Golden snub-nosed monkey,Gorilla,Greater bamboo lemur,Ma's night monkey,Macaque,Marmoset,Angola colobus,Black snub-nosed monkey,Bolivian squirrel monkey,Bonobo,Bushbaby,Capuchin,Chimpanzee,Coquerel's sifaka,Crab-eating macaque and Drill.

\section{Methods}

2.1.According to the structural characteristics ${ }^{[1]}$ of SARS-CoV-2 nucleic acid and the theory of nucleic acid codon,SARS-CoV-2 genomes were virtually segmented.The nucleic acid sequences were selected.

2.2.Using the principle of complementary base pairing ${ }^{[2]}$ and the sequences obtained by the method $\nabla 2.1 \rrbracket$,the antisense nucleic acid sequences were selected.

2.3.Highly similar sequences were selected from the sequences obtained by the methods $₫ 2.1$ and $2.2 \rrbracket$.

2.4. The antisense nucleic acid sequences and corresponding highly similar sequences obtained by the method $₫ 2.3 \rrbracket$ were compared with 44707 SARS-CoV-2 genomes. The unmatched sequences were selected.

2.5.The sequences obtained by the methods $₫ 2.4 \rrbracket$ were compared with human genome.Those sequences would be selected if they do not match successfully at the same time. 
2.6.The sequences obtained by the method $₫ 2.5 \llbracket$ were compared with 26 primates' genomes. Those sequences would be selected if they do not match successfully at the same time.

2.7.The nucleic acid sequences and highly similar sequences were selected through the sequences obtained by the methods $₫ 2$.6\.They were compared with 44707 SARS-CoV-2 genomes. The inhibition rate could be calculated.

\section{Results}

\subsection{The result of the initial screening.}

2288 sequences were screened by virtual segmentation of SARS-CoV-2 genomes. 2205 sequences were screened out while the antisense nucleic acid sequences and corresponding highly similar sequences compared with 44707 SARS-CoV-2 genomes.Compared with human genome, 1702 sequences were screened. While the 1702 sequences were compared with 26 primates' genomes, 181 sequences were screened.

\subsection{Inhibitory effects of the screening}

Eliminating the sequences that are very similar to each other, 19 sequences with over $95 \%$ matching rate and the number of bases is under 24 were screened out. 19 sequences and their highly similar sequences were matched with 44707 SARS-CoV-2 genomes. The combined matching rate was $100 \%$, which was also the inhibition rate of the corresponding 19 main antisense oligonucleotides sequences.

\subsection{Components of antisense oligonucleotides}

5'UUACACGAUAACCAGUAAAGACAU3'

5'UUAAUUGUGUACAAAAACUGCCAU3'

5'UUAACCUCUCUUCCGUGAAGUCAU3'

5'UUAACAAUGGUUGUACAU3'

5'CUAAAACAACACGAACGUCAU3'

5'UCAAUACUUGAGCACACUCAU3'

5'CUAAUAACGAGAUCCCAU3'

5'UUAAAACACCCUCUUGAACAACAU3'

5'CUAAGUCCGUGCCAGCAU3'

5'UUAAUUUGCGUGUUUCUUCUGCAU3' 
5'UCAGUGAAUACUGUGAAAUUCCAU3'

5'UUAAAUUCUUGGCAACCUCAU3'

5'UCAAUGGGCAAGCUUUGUCAU3'

5'CUAUAACGCAGCCUGUAAAAUCAU3'

5'UUAACAAAAUCGCCCGUCUGCCAU3'

5'UCAGUACACCAACAAUACCAGCAU3'

5'UUAAAAACCAGUGCGUGCCAU3'

5'UUAGUAACAAAGGCUGUCCACCAU3'

5'UUACGUAUGCAAACACCACAU3'

\section{Discussion}

4.1. In the application of this technique, it is necessary to pay attention to the problem of high similarity between sequences. Of the same length, the same direction ( $5^{\prime}-3^{\prime}$ or $\left.3^{\prime}-5^{\prime}\right)$, the same position and the same base,they are highly similar.In order to improve the reliability of the experimental results, the proportion of same bases is more than $90 \%$.

4.2.The reason for the comparison with the 26 primates' genomes is that there are still many undefined bases in human genome. Therefore, the use of this comparison method can minimize the probability of error.

4.3.In view of the current human understanding of the scope of the accuracy of electricity, uniqueness of the attributes, it is the best control tool.Therefore, the local charge of the organism directly affects the hydrogen bond charge distribution in the nucleic acid chain, the binding site of the similar charge nucleic acid chain can work normally, and the different charge will interfere, which leads to the failure of synthesis.As a result, nucleic acid compounds are made differently in different parts of the organism because of the different distribution of electric charges.

\section{Declarations}

\section{Data availability}

All data and materials are available on the Open Science Framework at https://osf.io/rew37/quickfiles. Publish a preprint on Research Square(https://www.researchsquare.com/article/rs-479520/v1) and https://europepmc.org/article/PPR/PPR320094. 
Code availability

All code used to produce analyses for all studies is available on the Open Science Framework at https://osf.io/rew37/quickfiles.

\section{Acknowledgements}

This article is dedicated to my mother, thank her for lefting me precious wisdom, deeply arousing the conscience of my soul!

\section{Author information}

\section{Affiliations}

Fujian Animal Husbandry General Station,Fuzhou,Fujian,China

Hua wei SHEN,Professor-level senior livestock technician

\section{Contributions}

This article was written by Hua wei SHEN alone.

\section{Corresponding authors}

Correspondence to Hua wei SHEN.

\section{Ethics declarations}

Competing interests

The authors declare no competing interests.

\section{References}

[1].Huawei SHEN Research on Simulative Technology in Destroying the viral weapons, publisher:Blurb,Jun 07,2017 p8-11 and p36-41,eBook link:https://www.blurb.com/b/7987986

[2].E.Chargaff, R.Lipshitz, C.Green.Composition of the deoxypentose nucleic acids of four genera of seaurchin . J Biol Chem. 1952;195 (1): 155-160.

\section{Supplementary Files}

This is a list of supplementary files associated with this preprint. Click to download.

- initialscreeningsequences.xls 
- matchingrate.xls

- SARSCoV2accessionnumber.xls

- Theresultcomparedwith26primatesgenomes.xls

- Theresultcomparedwithhumangenome.xls 